\title{
Annie Ernaux: l'écriture du "don reversé"
}

\author{
Philippe Vilain \\ Université Paris III - Sorbonne Nouvelle
}

Lavant dernier paragraphe de Passion simple s'achève sur ce constat: "J'ai
seulement rendu en mots [...] ce que son existence, par elle seule, m'a apporté. Une sorte de don reversé" (PS, 76-77).

Cette question du don mérite d'être discutée. En effet, si l'existence de A., l'amant, semble avoir été déterminante dans la vie de la narratrice, rien ne montre que celui-ci ait été responsable de sa propre influence. La nature même du prétendu don que la narratrice semble vouloir à toute force lui attribuer n'est d'ailleurs jamais explicitement mentionné. Au contraire même, elle recense ses manquement: par prudence d'homme marié, A. n'envoie pas de lettres, $(P S, 29)$ n'offre ni fleurs ni cadeau et manifeste peu d'attention à l'égard de la narratrice. ${ }^{1}$ Il semble que ce don relève davantage d'une construction imaginaire de la part de la narratrice que d'une volonté réelle de A. Pour compenser ses frustrations et se prouver à elle-même l'amour de A., la narratrice cherche obstinément les signes indiscernables de la passion:

"Il me fait cadeau de son désir." J'enregistrais avidement les phrases que je prenais pour des signes de jalousie, seule preuve pour moi de son amour. Après quelque temps je m'apercevais que "est-ce que pars pour Noël ?" n'était qu'une question banale ou pratique, pour prévoir ou non un rendezvous, nullement une manière détournée de savoir si j'allais au ski avec quelqu'un (peut-être même souhaitait-il que je parte afin de voir une autre femme ?). Je me demandais souvent ce que signifiaient pour lui ces aprèsmidi passés à faire l'amour. Sans doute rien d'autre que cela justement, faire l'amour. De toute façon, il était inutile de chercher des raisons supplémentaires, je ne serais jamais sûre que d'une chose : son désir ou son absence de désir (PS, 34-35). 
La répétition de la première personne associée aux verbes enregistrer, s'apercevoir, se demander renforce très nettement l'incertitude de la narratrice. Que A. fasse cadeau de son désir reste ici une interprétation de la narratrice.

Etudier le don dans Passion simple revient donc à poser la question de la générosité de l'amant. A. ne donne pas, il est. Ce qu'il donne, par conséquent, il le donne malgré lui : un peu de sa présence, mais comme le ferait tout autre être humain. N'est-ce pas le propre d'être que de donner de sa présence ? Il n'y a de don de soi uniquement dans la mesure où le don engage une volonté du donataire. Dans cette optique, peut-on raisonnablement définir le désir de A. comme un don ? Sauf à considérer le désir et l'acte sexuel dans une acception catholique, où sont prônées les valeurs du don, du partage, de fidélité et d'union, on ne saurait répondre à cette question que par la négative. Même si le titre Passion simple joue sur l'ambiguité du sens - christique - de Passion, même si l'attitude de la narratrice accomplissant une sorte de pèlerinage amoureux à Padoue, apposant une photo de $\mathrm{A}$. sur une pierre, faisant trois voeux en entrant dans les églises, emprunte à l'attitude religieuse, par sa forme brève et son refus du romanesque, Passion simple est avant tout un récit de la transgression, un récit profane qui désacralise ${ }^{2}$ tout à la fois la Passion et une certaine idée de la littérature.

Par ailleurs, l'interprétation du don repose dans ce texte sur un présupposé idéaliste selon lequel le don est dégagé de toute intention provenant du donataire. Or, avant que d'être un vouloir donner - ce qu'il ne réussit pas toujours à être - le désir n'est d'abord qu'un égoïste vouloir posséder; dans cette mesure l'association du désir au cadeau parait ici absolument contestable. La nuance est fondamental: le vouloir donner est orienté vers l'autre, le vouloir posséder retourné vers soi.

La question du "don reversé" se révèle plus profonde encore. Il serait naïf de croire que la narratrice reverse le don de A. - nous avons vu que le don impliquait une volonté, un engagement -, nuance là encore: dans la mesure où le don est une construction imaginaire de la narratrice, celle-ci reverse donc ce qu'elle a cru que A. lui donnait. Mais après tout peu importe cette dernière nuance, seul importe comment la narratrice reverse. Et plutôt que de restreindre cette étude à Passion simple, appliquons-là aux récits immédiatement antérieurs à ce dernier. Tout autant qu'à l'amant de Passion simple, le "don reversé" peut tout aussi bien s'adresser au père ou à la mère, respectivement décrits dans La Place et Une Femme. La question du reversement, dont l'objectif est de rendre en mots une existence, fût-ce celle d'un parent ou d'un amant- demeure au centre de l'oeuvre d'Annie Ernaux et le projet sous-jacent sensiblement identique dans ces trois récits autorise à effectuer le rapprochement.

Il reste à saisir le sens effectif de ce don, à définir son rôle et sa fonction au niveau de l'écriture. Comprendre la circulation du don dans ces récits, c'est d'abord identifier le donataire du donateur, avant de mettre en évidence les procédés scripturaux par lesquels 
s'opère le reversement.

\section{Lien familial / déchirure sociale}

Tandis que les premiers romans, Les Armoires vides et Ce qu'ils disent ou rien, mettaient en scène un conflit à l'intérieur duquel les narratrices, déchirées entre leur milieu familial et le monde scolaire, rejetaient leur culture d'origine, les récits, La Place et Une Femme, ne s'attardent ni sur les raisons ni sur les tensions de ce conflit, mais s'efforcent au contraire de remettre au jour les liens qui unissait Annie Ernaux à ses parents. Le don s'inscrit, en première instance, dans le sens naturel de la filiation; les parents se trouvent à l'origine de la donation. Contraints très jeunes d'abandonner leur scolarité, les parents incitent leur fille à poursuivre ses études au détriment de l'image négative que renvoie une telle situation auprès de la clientèle de l'épicerie: au milieu des années cinquante, une fille de 17 ans qui ne travaillait pas s'exposait à la critique et risquait d'être considérée comme "une paresseuse" $(L P, 81)$. Permettre à leur fille de réussir en lui assurant les conditions matérielles suffisantes, tel est le désir des parents prêts à consentir d'importants sacrifices financiers pour que "la gosse (ne soit) privée de rien" (LP, 56).

De La Place à Une Femme, la générosité des parents se fait écho insistant: le père offre ses économies pour aider le jeune couple, la mère aime "donner à tous plus que recevoir" $(U F, 105)$. La plupart du temps ces dons sont associés à des actes ou bien matérialisés par du concret, de l'utile et du nécessaire (nourriture, vêtements, argent). Certains passages soulignent néanmoins un autre aspect de la générosité du père caractérisée par un don de soi: "Il me conduisait de la maison à l'école" $(U F, 112)$. Ce souvenir, répété par l'oncle au moment de l'inhumation, signale, par son occurrence, l'extrême importance accordée à un fait en apparence insignifiant mais dont le père devait secrètement se glorifier, signe de son entier dévouement pour sa fille.

C'est un élément corporel - la main — qui, dans La Place, est chargé de refigurer le don du père ainsi que le temps de la complicité heureuse : "Il me tenait par la main," "Il m'emmenait par la main" $(U F, 50)$, "Il me conduit par la main" $(U F, 45)$. Loin de figurer de façon éparse, la main intervient chaque fois à un moment clef du récit et apparaît de manière prégnante, elle se propage par un effet de redoublement lexical, de contamination thématique, créant ainsi une sorte d'intertextualité obsessionnelle qui englue l'image de la main dans le tissu scriptural des récit. ${ }^{3}$ Dans ces nombreuses répétitions le pronom personnel désignant les parents est généralement sujet de phrase (Il me ou elle me $e^{4}$ ), celui désignant la fille, et enchâssant directement le pronom personnel des parents, est, à l'inverse, objet. Il s'agit pour l'auteur de mettre l'accent sur le lien familial; ici, l'ordonnance syntaxique des pronoms personnels indique la domination parentale à travers laquelle la verticalité de la descendance est reflet de la circulation du don. Tout se passe comme si les marques de personne, en s'entrecroisant elles-mêmes, cherchaient la proximité d'un fairecorps verbal et réalisaient le collage du il-elle parental sur le je de la narratrice. L'écriture 
rassemble, photographie l'union reconstituée: "avec mon père, j'ai monté le grand escalier de la mairie" $(L P, 111)$. Cette montée pouvant être interprétée de manière psychanalytique comme une forme de remariage civil que l'écriture officialiserait.

Point nodal de la réunion du couple, la main figure en même temps le divorce social. En effet, le père en conduisant sa fille à la bibliothèque, située dans la mairie, en lui donnant accès au livre, conditionne à son insu une rupture inévitable. Pour cette raison, il n'est pas anodin que "le serrement de mains," dans l'incipit de La Place, soit emblématique de la rupture avec le milieu d'origine. Si l'obtention du CAPES est l'aboutissement du projet parental, il traduit aussi le passage de la classe dominée à la classe dominante, le passage du monde populaire, culturellement et économiquement précaire, au monde bourgeois, relativement dominant par le savoir.

Transmission d'un savoir, la poignée de mains concrétise aussi la réussite sociale représentée par la cérémonie d'ouverture du CAPES, très solennelle, avec son cortège d'éloges: "Madame, je vous félicite." Les autres ont répété "je vous félicite" et m'ont serré la main." C'est la transmission de la culture bourgeoise qui est donnée à lire entre ces lignes, la nouvelle orientation sociale de l'auteur. La fille lâche définitivement, symboliquement, la main de ses parents, et, ce franchissement baptismal efface d'un trait l'héritage du monde d'en bas. Par tout un jeu intertextuel de symétries formelles, le récit se focalise sur les mains de la caissière pour clôturer le texte. Après la réussite du concours, les dernières lignes proposent en quelque sorte l'échec du professeur dans son rôle d'éducateur. Il faut lire dans la résignation de la caissière, dans sa gêne, l'acceptation de son sort (le geste main droite-main gauche est devenu un geste automatique que la caissière reconduira toute sa vie), l'attitude d'une dominée en écho à la situation du père retiré de l'école à l'âge de treize ans pour travailler dans une ferme. En creux de La Place, il y a une dénonciation évidente du système scolaire qui ne parvient pas à réduire les inégalités socioculturelles de départ. Cette dernière scène exprime le sentiment de culpabilité et de trahison éprouvé par l'auteur: l'ancienne élève — socialement le double du père — n'a pas été sauvé par le professeur qui pourtant était du même milieu qu'elle. En ne se souvenant ni de sa classe, ni de son nom, le professeur signifie son échec.

La main joue donc thématiquement d'un bout à l'autre du récit, traverse le texte de part en part pour relier implicitement la réussite sociale à l'échec social, la fille au père, le jevivant au il-mort: cette-main-que-mon-père-tenait-autrefois-écrit-maintenant-sur-mon-père. Tout à la fois, élément fondamental de cette passation des pouvoirs et métonymie du labeur de l'écrivain, la main réalise l'accord avec les parents pour qui le sens de travailler signifiait avant tout "travailler de ses mains" ( $L P, 81)$. L'écriture, en permettant la rencontre posthume, reconduit les parents au chevet d'une réunion à la fois familiale et littéraire. Dès lors, la circulation du don et le sens de la transmission s'inversent pour faire glisser ostensiblement le "on n'avait plus rien à se dire" $(L P, 84)$ au je-vais-écrire-sur-mon-père. Cette textualisation du silence pose la question fondamentale de la fonction de l'écriture: 
"Est-ce qu'écrire n'est pas une façon de donner ?" $(U F, 106)$.

\section{Filiation et appartenance sociale}

A la différence de Denise Lesur et d'Anne, les narratrices des Armoires vides et de Ce $q u$ 'ils disent ou rien qui se déguisaient derrière les masques de la fiction sous un patronyme de substitution, les narratrices de La Place et Une Femme s'assument comme génitrices des textes. Le contact est ainsi rétabli entre l'auteur et les narratrices-personnages qui ont pour seule et unique identité, Annie Ernaux. Ces coordonnées intimes tissent en quelque sorte le pacte autobiographique des récits. L'affirmation du lien familial est d'ailleurs nettement renforcée: "Je n'ai vu qu'une seule fois mon grand-père, à l'hospice où il devait mourir trois mois après. Mon père m'a menée par la main à travers deux rangées de lits" $(U F, 25)$.

Cette première rencontre s'avère d'autant plus touchante qu'elle est aussi la dernière, l'ultime confrontation des trois générations de la lignée paternelle. Cette scène est ainsi l'occasion pour l'auteur de retracer son destin familial et de sceller son propre enracinement social:

L'histoire commence quelques mois avant le vingtième siècle, dans un village du pays de Caux, à vingt-cinq kilomètres de la mer. Ceux qui n'avaient pas de terre se louaient chez les gros fermiers de la région. Mon grand-père travaillait donc dans une ferme comme charretier $(U F, 24-25)$.

et, dans Une Femme: "Mon grand-père, charretier dans une ferme, et ma grand-mère, tisserande à domicile."

La petite-fille revendique ses origines populaires et accepte sa filiation authentique en s'inscrivant dans cette généalogie, désormais sans répudiation du familial. Annie Ernaux écrit sa propre préhistoire, et avec celle-ci tout le patrimoine familial, culturel, dont elle a hérité et qu'elle transmet par l'écritur: "J'ai fini de mettre au jour l'héritage" (UF, 111).

En ce sens, il est possible de parler de "don reversé," l'écriture s'assigne comme perspective d'exposer la trajectoire sociale des parents, leur culture, de rassembler leurs paroles, leurs gestes, leurs goûts, "les faits marquants de (leur) vie, tous les signes objectifs de (leur) existence." C'est, cependant, à un tout autre niveau que joue ce type de reversement. Il s'agit de prendre en charge tout un savoir populaire cristallisé dans la personne du père et de la mère, épicentres autour desquels gravite le sens du don. Les récits ernaliens réinscrivent la vie des parents en la décalant et en la dépassant. A travers ses parents, Annie Ernaux met en scène un processus d'écriture qui s'apparente à la méthode ethnologique, fondée sur la recherche des micro-structures signifiantes de la société des années cinquante, d'une gestus de classe - "tous les gestes qui s'accommodent de la pauvreté" $(U F, 26)$ - qui, enfant, était aussi la sienne.

En effet, autour de l'image du corps des parents, Annie Ernaux désigne un mode de 
socialité qu'elle a aussi partagé ${ }^{5}$ tout ce qui entre dans la structure globale d'un rapport au monde populaire: l'obsession et le caractère sacré de la nourriture ("Me voir laisser de la nourriture dans l'assiette lui faisait deuil. On aurait pu ranger la sienne sans la laver" $L P$, 68 ), l'aspect festif des repas de noce, de communion et d'inhumation; le rapport au vestimentaire, le père est décrit portant une casquette et un bleu de travail pendant la semaine et le dimanche en costume, "un ensemble, pantalon foncé, veste claire sur une chemise et une cravate" $(U F, 55) ;$ l'hygiène, le père se rase trois fois par semaine, face à la glace fixée au-dessus de l'évier de la cuisine, refusant obstinément d'utiliser le cabinet de toilettes installé à l'étage, il dort "toujours avec sa chemise et son tricot de corps" ( $L P$, 69); la méfiance par rapport aux sciences médicales, les croyances et superstitions dans les remèdes de grand-mère : "Pour chasser (les vers), on cousait à l'intérieur de la chemise, près du nombril, une petite bourse remplie d'ail." 6

Derrière ce rituel du corps et ses connotations sociales se reflètent l'épaisseur d'un déjàlà, les marques de la communauté paysanne et ouvrière de l'époque. Annie Ernaux dresse l'inventaire, emmagasine les images paternelle, non pas seulement pour restituer sa propre mémoire mais une mémoire collective plus profonde; de sorte que les récits ne suivent plus simplement une ligne de vie chronologique, mais creuse une épaisseur stratifiée où les images liés aux parents et les souvenirs des années cinquante s'inscrivent en surimpression, comme un palimpseste. Grattant mot après mot le crépi de sa mémoire, c'est une autre figure des parents que l'auteur redécouvre, non plus uniquement un singulier il ou elle mais un pluriel ils. Le don se construit ainsi au fil des récits en introduisant cette idée d'altérité: la figure des parents se trouve dissoute, "transpersonnalisée," dans une figure générale, universelle. ${ }^{7}$ L'altérité est une matière objective permettant d'étendre la recherche scripturale à l'ensemble d'une communauté, de l'élargir à un projet de type ethnographique. C'est précisément dans la revendication de son appartenance sociale, dans ce partage de tout le paradigme de quotidienneté des parents que tient la signification profonde de ce reversement: témoigner à la place des parents mais, plus profondément, à la place de tous ceux qui ne le peuvent pas, continuer la transmission d'une culture inhumée puis déterrée et remise en scène par l'écriture. Plus intéressant encore, sera de montrer un jeu de miroir entre les procédés d'écriture et la thématique de la donation. Il s'agit d'étudier la matérialité scripturale du don, de voir comment cette donation reversée se répercute un niveau de l'écriture.

\section{Réinstaurer un dialogue}

Si Annie Ernaux refuse catégoriquement d'annexer la question d'une écriture de soi comme dialogue à son oeuvre, ce refus demande à être examiné en profondeur. Alors qu'une première lecture des textes tendrait à confirmer l'opinion de l'auteur, une seconde, effectuée dans la perspective de ce débat, apporte une réponse plus nuancée. Certes, dans l'oeuvre d'Annie Ernaux, le dialogue n'apparaît jamais de manière directe, explicite, au sens où le présente Nathalie Sarraute dans Enfance, à savoir un échange marqué par des répliques abondantes voulant donner l'illusion d'une conversation réelle entre un je et un 
$t u$, produits d'une seule et même conscience. Evitant de rester prisonnière des contingences autobiographiques, voire égotistes, Annie Ernaux se détourne de la psychologie individuelle en mettant en scène, non un je introspectif, mais un je traversé par des expériences ordinaires (la mort d'un parent, l'inégalité sociale, la passion), un je miroir d'une société, analysé sous un éclairage socio-historique. Elle semble aborder l'écriture comme une recherche dialogique de soi à travers les autres, un questionnement de l'individuel à travers le collectif.

Dans La Place et Une Femme, un dialogue s'instaure entre l'auteur et le narrateur. Dialogue implicite qui se présente sous la forme d'une recherche scripturale intégrée à la quête des parents. A la manière d'un journal de fouilles, ces récits proposent une mise au jour progressive d'existences, d'expériences vécues et de réflexions sur l'écriture. Ces réflexions, traversées par une interrogation plus générale sur la manière d'écrire, le souci de la sincérité, le doute, sont à l'évidence les expressions sous-jacentes d'un dialogue intérieur à l'intérieur duquel Annie Ernaux propose l'alternance entre le récit et le commentaire, entre le souvenir et le jugement sur ce souvenir, participant en cela de la structure du "double registre" analysée par Jean Rousset dans Formes et significations. Ainsi, par exemple, dans Une Femme:

En écrivant, je vois tantôt la "bonne" mère, tantôt la "mauvaise". Pour échapper à ce balancement venu du plus loin de l'enfance, j'essaie de décrire et d'expliquer comme s'il s'agissait d'une autre mère et d'une fille qui ne serait pas moi $(U F, 62)$.

L'auteur semble partagé entre écrire sur ses parents et se regarder écrire. D'où cette forme dialogique imposée par une écriture de ruptures et un rythme binaire de reconstruction entre le récit du passé par le narrateur et les interventions de l'auteur au présent. Sans masquer le fonctionnement de ses récits, l'auteur engage un véritable dialogue avec son enfance et ses parents, avec ses souvenirs; il médite, en quelque sorte, et pour reprendre le mot de Balzac, "les brosses à la main."

Des romans aux récits, les textes d'Annie Ernaux se questionnent et se répondent entre eux, creusant indéfiniment la même matière spatio-temporelle - l'enfance après la Seconde Guerre, le café-épicerie des parents, le pensionnat religieux d'Yvetot -, les mêmes motifs - l'aliénation sociale, le changement de classe sociale. A travers leurs obsessions et la réitération de motifs sans cesse reprisés, ses textes sont l'objet d'un dialogue intertextuel qui traversent l'univers de l'oeuvre. Ecrire sur soi implique de puiser sans cesse dans le puits de ses origines, de retracer le cheminement d'un destin familial. La petite-fille revendique ses origines populaires en s'inscrivant dans cette généalogie. C'est dans la revendication de sa propre appartenance sociale, dans ce partage de tout le paradigme de quotidienneté des parents que l'auteur renoue la signification profonde du dialogue avec ses parents: La Place apparaît comme la fin d'une conversation entre le père et sa fille, le dernier mot en réponse à l'amour du père: "J'écris peut-être parce qu'on n'avait 
plus rien à se dire" $(L P, 84)$.

Une Femme apparaît comme un enfantement de la mère par l'écriture, une sorte de don reversé:

"Il me semble maintenant que j'écris sur ma mère pour, à mon tour, la mettre au monde" $(U F, 43)$. Là encore, l'auteur restaure le dialogue avec les parents.

L'oeuvre d'Annie Ernaux, on le voit, malgré les réticences de l'auteur lui-même, a non seulement partie liée avec la problématique posée par l'écriture de soi comme dialogue, mais l'excède quelque peu. Rattachée à l'autobiographie, l'originalité de cette oeuvre est de présenter un dialogue intérieur activant les mécanismes d'un type d'écriture de recherche et un dialogue transpersonnel renouvelant la manière de penser le sujet au centre de la littérature et de l'Histoire. A quel genre autobiographique appartiennent La Place et Une Femme? Ernaux emploie le terme de auto-ethnographie pour qualifier son entreprise. Ses récits ne sont ni des biographies, ni des romans, mais "peut-être quelque chose entre la littérature, la sociologie et l'histoire" $(U F, 106)$. Aux confluents de disciplines aussi distinctes que solidaires, les récits d'Annie Ernaux brouillent les classifications ordinaires sans jamais s'inscrire dans un genre précis ou se laisser circonscrire par lui. Cette rare promiscuité générique ne fait que consacrer une rupture avec l'autobiographie traditionnelle. Il semble, précisément, que ce brassage - melting pot des formes possibles de l'écriture du moi - soit, exposé sous sa forme du questionnement, le lieu d'un véritable dialogue sur la littérature autobiographique.

\section{Ecriture neutre ou écriture engagée ?}

Dans un milieu où la culture littéraire est jugée d'avance inutile, antinomique même du travail, l'écriture apparaît au centre d'une problématique de classe sociale, celle-ci étant considérée comme une activité de luxe. Dans La Place et Une Femme, il n'est plus question que l'écriture incisive et violente serve d'instrument à la dénonciation de la classe d'origine, comme c'était le cas dans les premiers romans, il s'agit désormais d'écrire autrement, en s'interrogeant sur le mode d'écriture à adopter: quelle écriture pour parler de mes parents issus d'un milieu populaire? Fidèle aux principes du récit-vrai, Annie Ernaux doit également trouver tout à la fois un style susceptible de se tenir au plus près du référentiel ou de la vraisemblance et surtout une esthétique capable de se rapprocher des valeurs couramment attribuées à la classe dominée -la simplicité, la sincérité, l'utilité, effacement de soi —, fondées sur "l'affirmation de la continuité de l'art et de la vie." ${ }^{8}$ La question du reversement scriptural trouve ici sa justification profonde dans ces valeurs que Pierre Bourdieu, dans La Distinction, assigne au mode et à l'esthétique populaires.

Si La Place présente un condensé des Armoires vides, ce texte ne marque pas moins une rupture esthétique très nette dans l'oeuvre d'Annie Ernaux, qui coïncide avec l'abandon et le rejet de la fiction. ${ }^{9}$ La finalité scripturale converge désormais dans le sens d'un refus de la littérature bourgeoise. Entendons par littérature bourgeoise, sans trahir la vision d'Annie 
Ernaux, une littérature qui reproduirait - implicitement ou explicitement - les valeurs du monde bourgeois (primat du moi et intérêt porté à la vie domestique), et qui aurait pour destinataire le lectorat dominant, détenteur du capital économique et culturel ; une littérature identifiée au romanesque qui aurait pour fonction d'enchanter le monde. C'est pourquoi l'écriture plate parait s'imposer d'elle-même, spontanément. "Pour rendre compte d'une vie soumise à la nécessité," l'auteur retrouve une langue primitive, réduite à l'essentiel, et prend soin d'éviter toutes les ressorts traditionnels du cérémonial romanesque. Les guillemets placés sur "passionnant" et "émouvant" $(L P, 24)$ soulignent d'ailleurs implicitement tout autant le souci de se tenir à l'écart de l'affect ou du pathos romanesque que le désir de rompre avec un bien-écrire, avec un écrire-correctement. Ennaux refuse la plupart des usages qui régissent l'écriture de fiction dépensière et gratuite pourtant sensible dans les Armoires vides ou de La Femme gelée - inflation lyrique, descriptions, tentation poétique. L'écriture ernalienne bannit désormais toute "recherche de style" que les parents auraient ressentie comme une manière de les tenir à distance $(L P, 90)$, tous les procédés qui risqueraient de travestir l'image, de magnifier le vécu des parents et, de les perdre définitivement dans une autre histoire, sous le masque d'une identité fictive. Le ton du constat permet la mise en scène le réel mais aussi la transposition des valeurs dominées dans le registre scriptural réservé au monde dominant. Ce qui a pour effet direct de produire un nivellement des valeurs dominantes/dominées et de reconstruire une continuité entre l'art et la vie.

Dans ces récits, l'affirmation de l'appartenance sociale à la classe d'origine est renforcée par le refus de complicité avec le lecteur. Ernaux montre ses parents sans regard critique, sans les placer dans la culture dominée, mais en les transcrivant tels qu'ils étaient. Son projet fait nettement apparaître une dénonciation de l'aliénation ouvrière, il tend à redonner la dignité à ses parents, à transformer l'indignité en dignité. C'est du reste l'une des contradictions centrales de La Place, car s'il n'est évidemment pas question d'une réhabilitation du père, l'auteur lui assigne cependant une série de caractérisants relativement positifs : à plusieurs reprises, la fille vante le courage de son père ("Il faisait deux kilomètres à pied pour atteindre l'école" $(L P, 29)$; "Les heures de travail ne se comptaient pas" $L P, 32$ ), ses mérites ("Il a réussi à savoir lire et écrire sans faute. Il aimait apprendre" $L P, 29$ ), ou bien lui trouve des circonstances atténuantes ("Mon grand-père l'a retiré de l'école pour le placer dans la même ferme que lui. On ne pouvait plus le nourrir à rien faire" $L P, 29-30$ ). Cette série de caractérisant positifs marque ici à la fois l'engagement de l'écrivain-intellectuel aux côtés du père mais également au côté de l'ensemble de la classe dominée.

L'objectivité, c'est une évidence, recèle en elle-même ses propres limites. Sa pertinence est discutable: peut-on prétendre à l'objectivité totale? L'important pour Annie Ernaux n'est pas tant de réussir à être totalement objective que de tendre sincèrement à l'objectivité. Il s'agit plutôt d'une règle d'écriture qu'un gage assuré de réussite. La littérature décrit rarement les gens de la classe inférieure de façon juste et objective, ces derniers sont le plus 
souvent l'objet d'une présentation hyperbolique: par excès, c'est le réalisme populiste par où une vision embellie et valorisante des classes ouvrière et paysanne trahit tout autant la présence des signes d'une connivence culturelle avec le monde dominant; par défaut, c'est le misérabilisme de Zola où se mêlent tristesse et infériorisation, noirceur et aliénation. Traditionnellement, du populisme au misérabilisme, la classe dite "inférieure" n'accède à la description littéraire qu'en s'annulant dans l'exagération, l'hyperbolisation et l'exotisme social, déformée dans un trop Beau ou un trop Laid. A défaut de l'annihiler complètement, l'écriture réduit, au contraire, l'écart de manière spectaculaire et interdit, du fait de sa neutralité, tout jugement moral susceptible se glisser derrière la vision de l'auteur.

Cette inclination vers l'objectivité se manifeste encore par la mise en scène d'un processus d'écriture qui s'apparente à la recherche d'une méthode. Valorisation de l'éclatement de la forme au détriment de la sensiblerie romanesque et des petites facilités mnésiques qui, depuis Proust, ont largement tendance à "fleurir" dans les romans contemporain:

Je ne pouvais pas compter sur la réminiscence, dans le grincement de la sonnette d'un vieux magasin, l'odeur de melon trop mûr, je ne retrouve que moi-même, et mes étés de vacances, à $Y$... La couleur du ciel, les reflets des peupliers dans l'Oise toute proche, n'avaient rien à m'apprendre" ( $L P$, $100)$.

Toute l'originalité de l'écriture ernalienne réside dans cet éclatement des procédés romanesques conventionnels, originalité caractérisée par le refus du style, l'absence de transition facile, l'exposé du fait brut sans explication, le discontinu, l'insert du patois, italiques, références de bas de page, parenthèses et guillemets; ces différentes pratiques convergent vers une forme susceptible de rendre compte de la réalité vécue, d'imiter le côté défait d'une l'écriture épistolaire et plate tournée vers l'utile et l'essentiel: "Aucune poésie du souvenir, pas de dérision jubilante. L'écriture plate me vient naturellement, celle-là même que j'utilisais en écrivant autrefois à mes parents pour leur dire les nouvelles essentielles" $(L P, 24)$.

Désormais l'accent n'est plus seulement porté sur l'histoire elle-même mais, plus subtilement, sur la façon d'écrire l'histoire. Peut-être faut-il voir dans l'implosion des procédures ordinaires de signifiance, dans l'explosion de la textualité, l'introduction des valeurs de la classe dominée réalisant leur propre révolution artistique et culturelle à l'intérieur du monde littéraire dominant? Economie et dépouillement s'avèrent être les procédés moteurs de cette pénétration dans le monde dominant que représente "l'écriture bourgeoise." Le projet sous-jacent vise à démonter les ressorts fictionnels, à montrer le rétrécissement du champ scriptural dans sa dimension créatrice et à mettre à nu le rapprochement de sa conception. Dans la mesure où l'ensemble des procédés scripturaux occupent ici une fonction quasi sociale (renforcer et redoubler l'oppression économique 
dont furent victimes les parents), la tâche de l'écrivain-intellectuel, dans la lignée marxiste de la lutte des classes, semble être "d'accentuer la décomposition de la conscience bourgeoise."10 Cette forme de degré zéro de l'écriture semble justement marquer l'engagement de l'intellectuel, le désir de pactiser avec ses parents et sa classe d'origine. Débarrassée de ses scories esthétisantes, réduite par l'épure à sa plus simple expression stylistique, l'écriture reverse aux parents leurs propres dons. Les économies que faisaient les parents pour satisfaire aux besoins de leur fille sont maintenant transférées dans le texte. En se textualisant, les mots se rendent pratiquement invisibles, restent enfouis derrière la chose qu'ils désignent; ils ne sont jamais vus par le lecteur comme des mots, mais comme une vitre que celui-ci pourrait traverser. Cette textualisation permet, par conséquent, d'articuler et d'entrelacer dans le même champ la pratique littéraire avec l'élément économique, soit de relier implicitement la littérature à la vie. Réalisme extrême et désir de transparence qui travaillent de concert avec le souci de replacer les parents, objectivement et dignement, dans cet espace-temps reconfiguré qu'est l'écriture; même si au-delà de la séparation remise en mots, règne l'impossible et tragique reversement d'un don qui ne sera jamais lu.

\section{Abréviations}

PS: Passion simple, Gallimard, collection folio, $\mathrm{n}^{\circ} 2545$.

UF: Une Femme, Gallimard, collection folio, $\mathrm{n}^{\circ} 2121$.

$L P$ : La Place, Gallimard, collection folio, $\mathrm{n}^{\circ} 1722$.

\section{Notes}

${ }^{1} P S, 34 . "[\ldots]$ je pensais aux attentions que lui ne jugeait pas nécessaire d'avoir à mon égard [...]."

${ }^{2}$ Michèle Bacholle. "Passion simple d'Annie Ernaux : vers une désacralisation de la société française?" Dalhousie French Studies, automne 1996. Concernant le sacré dans Passion simple, lire également Philippe Vilain. L'Etreinte, roman (Gallimard, octobre 1997, p. 58), qui retrace la relation amoureuse d'un jeune homme avec la narratrice de Passion simple. De cet homme A., de cette passion matérialisée par un livre, va naître la jalousie du narrateur de L'Etreinte. Alors que Passion simple proposait une description de la passion, L'Etreinte propose symétriquement une analyse des mécanismes de la jalousie.

${ }^{3}$ Un recensement du vocabulaire concernant les diverses parties du corps, étendu à l'ensemble de l'oeuvre d'Annie Ernaux, montre que la main est la partie la plus énoncée (203 fois: 22 fois dans La Place et 20 fois dans Une Femme), cf. Philippe Vilain, "Le sexe et la honte dans l'oeuvre d'Annie Ernaux," Roman 20-50 (Revue d'étude du roman du XXè siècle, Université de Lille III), $\mathrm{n}^{\circ} 24$ (décembre 1997): 149-164.

${ }^{4}$ Ibid., p. 51 : "Elle m'offrait des jouets et des livres à la moindre occasion". 
${ }^{5}$ La Place, p. 24 : "Je rassemblerai les paroles, les gestes, les goûts de mon père, les faits marquants de sa vie, tous les signes objectifs d'une existence que j'ai aussi partagée". Dessein qui confirme l'intention de départ dont le titre n'était pas La Place mais Eléments pour une ethnographie familiale.

${ }^{6}$ Ibid., p. 28. Pour plus de précisions, lire le relevé effectué par Marie-France Savéan dans son livre, La Place et Une Femme d'Annie Ernaux. Paris : Gallimard, collection Foliothèque. (en particulier le chapitre III, "Une étude sociologique des milieux populaires").

${ }^{7}$ Ibid., p. 100-101: "dans des êtres anonymes rencontrés n'importe où, porteurs à leur insu des signes de force ou d'humiliation de la réalité oubliée de sa condition". Ce thème - la recherche de soi ou d'un parent dans les autres - que nous retrouvons dans Journal du dehors, p. 106-107, sensiblement dans les mêmes termes : "D'autres fois, j'ai retrouvé des gestes et des phrases de ma mère dans une femme attendant à la caisse du supermarché. C'est donc au-dehors, dans les passagers du métro ou du R.E.R., les gens qui empruntent l'escalator des Galeries Lafayette et d'Auchan, qu'est déposée mon existence passée. Dans des individus anonymes qui ne soupçonnent pas qu'ils détiennent une part de mon histoire, dans des visages, des corps, que je ne revois jamais. Sans doute suis-je moi-même, dans la foule des rues et des magasins, porteuse de la vie des autres."

${ }^{8}$ Pierre Bourdieu. La Distinction: critique du jugement social (Paris: Les Editions de Minuit, 1985). Voir la première partie, chapitre 1 intitulé "Titres et quartiers de noblesse culturelle," p. 33: "Tout se passe comme si 1' "esthétique populaire" était fondée sur l'affirmation de la continuité de l'art et de la vie, qui implique la subordination de la forme à la fonction, ou, si l'on veut, sur le refus du refus qui est au principe même de l'esthétique savante, c'est-à-dire la coupure tranchée entre les dispositions ordinaires et la disposition proprement esthétique."

${ }^{9}$ La Place, p. 23: "j'ai commencé un roman dont mon père était le personnage principal. Sensation de dégoût au milieu du récit."

10 Roland Barthes par Roland Barthes (Editions du Seuil, collection "Ecrivains de toujours," 1975),

p. 67: "Il faut alors garder à l'image toute sa précision; cela veut dire qu'on feint volontairement de rester à l'intérieur de cette conscience et qu'on va la délabrer, l'affaisser, l'effondrer, sur place, comme on ferait d'un morceau de sucre en l'imbibant d'eau." Laurence Mall a analysé plus en détail ce rapport entre le style d'Annie Ernaux et l'oppression économique dans une excellente étude. "«Moins seule et moins factice:" la part autobiographique dans Une Femme d'Annie Ernaux," The French Review Vol. 69, n 1 (october 1995): 45-53. 